\title{
A test for the implementation-maintenance model of reappraisal
}

\author{
Christian Paret, Jessica Brenninkmeyer, Benjamin Meyer, Kenneth S. L. Yuen, Nina Gartmann, \\ Marie-Luise Mechias and Raffael Kalisch*
}

Institute for Systems Neuroscience, University Medical Center Hamburg-Eppendorf (UKE), Hamburg, Germany

Edited by:

Gilles Pourtois, University of Ghent, Belgium

Reviewed by:

David Sander, University of Geneva, Switzerland

Susanne Erk, Charite

Universitätsmedizin Berlin, Campus

Mitte, Germany

*Correspondence:

Raffael Kalisch, Institute for Systems Neuroscience, University Medical Center Hamburg-Eppendorf (UKE), Martinistraße 52, 20249 Hamburg, Germany.

e-mail: rkalisch@uke.uni-hamburg.de
Reappraisal has been defined as a conscious, deliberate change in the way an emotional stimulus is interpreted, initiated in order to change its emotion-eliciting character (Gross, 2002). Reappraisal can be used to down-regulate negative emotions, including anxiety (reviewed in Kalisch, 2009). There is currently a strong interest in identifying the cognitive processes and neural substrates that mediate reappraisal. We have recently proposed a model (termed implementation-maintenance model or IMMO) that conceptualizes reappraisal as a temporally extended, dynamic, and multi-componential process (Kalisch, 2009). A key tenet of IMMO is that reappraisal episodes are marked by an early phase of implementation that may comprise strategy selection and retrieval of reappraisal material into working memory, and a later phase of maintenance that may comprise working memory and performance monitoring processes. These should be supported by dissociable neural networks. We here show, using a detachment-from-threat paradigm and concurrent functional magnetic resonance imaging, that reappraisal-related brain activity shifts from left posterior to right anterior parts of the lateral frontal cortex during the course of a reappraisal episode. Our data provide first empirical evidence for the existence of two separable reappraisal stages. Implications for further model development are discussed.

Keywords: emotion regulation, reappraisal, detachment, distancing, fear, anxiety, prefrontal cortex

\section{INTRODUCTION}

Reappraisal modulates the evaluation of a stimulus in terms of its emotional-motivational meaning for the organism, a set of processes that is collectively termed appraisal. According to appraisal theorists, appraisal precedes the emotional response and is causal in its generation (is a "response-antecedent"; Roseman and Smith, 2001; Scherer, 2001). If one makes a simplifying assumption that the processing of an emotional stimulus consists in only one sequence of stimulus detection - appraisal response, this leads to the prediction that reappraisal must work at the appraisal stage and thus occur before the behavioral response is elicited (Gross, 1998). Reappraisal would thus be a quick and largely effortless process that lastingly changes the trajectory of the emotional response without the need for extended monitoring of reappraisal success (see Gross, 2002, and Richards, 2004, for reviews). At the neural level, this line of thinking has led to a model whereby reappraisal-related brain activity should be observable exclusively during early time points of an emotional episode (Goldin et al., 2008).

However, theorists have also argued for a more dynamic view of reappraisal that incorporates the idea of multiple, sequential "detection - appraisal - response" cycles (Gross and Thompson, 2007; Kalisch, 2009). This refinement is in congruence with modern appraisal theory which views appraisal as a recurrent process (Roseman and Smith, 2001; Scherer, 2001): Emotional stimulation is often temporally extended and varies over time; further, our own emotional reactions may change the situation and may also function as emotional stimuli in their own right. Hence, the appraisal process must continuously incorporate new information and integrate it in order to enable continuous response adjustments. For reappraisal, this highly dynamic nature of the appraisal process means that very often, if one desires to change one's emotional state into a specific (e.g., more positive) direction, one may also have to continuously adjust one's reappraisals (Kalisch, 2009). In addition, there may be situations where the emotional stimulation is rather monotonous and its essential meaning quickly appraised, but because the stimulus is so significant and strong we may have to make a continuous effort to overwrite our spontaneous and natural appraisals with the ever same reappraisal thoughts. This may be the case, for instance, when we experience extended pain or anticipate potential harm (anxiety).

To take these considerations into account, we have proposed a model (implementation-maintenance model or IMMO) which conjectures that the requirement for flexibility may result in a switching between operations that promote the implementation of a reappraisal strategy (that is, choosing between, and retrieving, potential reappraisals from memory) and those that promote the maintenance of a chosen strategy (that is, working memory). Maintenance processes must involve a component that monitors success in emotion regulation and can initiate new implementation activity or enhance maintenance efforts. Under normal circumstances, that is, with at least moderate reappraisal success, one can make a simplifying prediction that implementation processes should be predominant early during a reappraisal 
episode, while maintenance processes should prevail during later periods. Both sets of processes should be associated with distinct neural activation patterns (Kalisch, 2009).

Supporting this simple biphasic model, a meta-analysis of existing neuroimaging studies of reappraisal had indicated that early and late phases of reappraisal episodes are indeed supported by different neural substrates. Studies with comparatively short reappraisal episodes, which should be marked mainly by implementation efforts, preferentially activated left posterior lateral frontal cortex (LFC). By contrast, studies with comparatively long reappraisal episodes, where maintenance processes should increasingly come into play, preferentially activated right anterior LFC (Kalisch, 2009).

In the present experiment, we asked whether this pattern can be reproduced at the level of a group study. If so, this would be empirical evidence for the existence of an early and a late stage of reappraisal, at least within the context of the paradigm employed in this study. Specifically, we tested whether the major focus of reappraisal-related activation would shift across a reappraisal episode from left posterior to right anterior lateral frontal sites.

\section{MATERIALS AND METHODS OVERVIEW OVER DESIGN}

Anxiety was induced using a classical instructed fear paradigm (also known as "anticipatory anxiety") which consisted in forewarning subjects that they might receive a painful electric stimulus at any time during a 17.7-s trial (Threat condition, T). During a control condition (No-threat, NT), subjects were told they would not be stimulated during the trial. In a fully balanced, two by two factorial design, subjects either employed reappraisal (Reappraisal condition, R), or not (No-reappraisal comparison condition, NR). For details, see Figure 1 and the section below. For the Reappraisal condition, subjects were given a short self-statement and an associated visual imagery that both expressed a distanced, detached observer position. Subjects were supposed to bring up this reappraisal material at the beginning of a trial when cued, and then to mentally rehearse it throughout. Prior to the experiment, subjects had received some moderate training in retrieving and rehearsing the strategy. In the No-reappraisal condition, subjects were supposed to attend to the situation and to their emotional reactions but not to try to change them. In both conditions, subjects were eyes closed.

Four general considerations motivated our choice of design. First, a continuous threat of shock is a type of emotional stimulation that is both temporally extended and rather monotonous, the latter because its meaning to the organism is easily and quickly appraised and does not change over the course of a trial. It should thus leave enough time for observing potential spatio-temporal activation shifts while at the same time eliminating to some extent inter-trial and inter-individual variance in appraisal and reappraisal processes that might otherwise arise from a complicated or varying external situation (such as when stimulated by affective pictures or film clips). Both should facilitate the detection of common spatio-temporal activity patterns across subjects. Second, threat of pain is naturally and spontaneously appraised as relevant to the organism. Stimulus relevance is considered a major appraisal

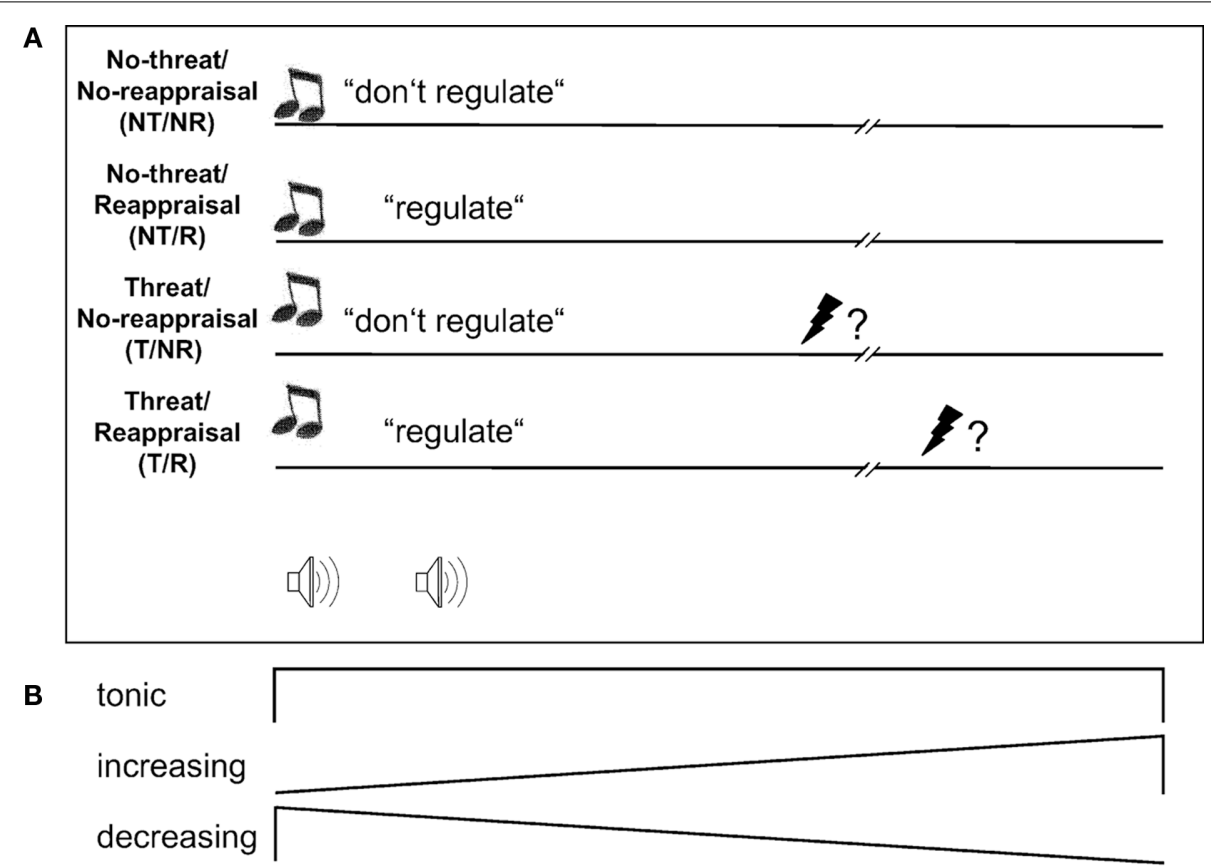

FIGURE 1 | Design. (A) At the onset of threat (T) trials, a high-pitch double-beep signaled subjects they might receive a painful electric stimulus to the hand at a probability of $25 \%$ at any time during the trial, which lasted $17.7 \mathrm{~s}$. At the onset of no-threat (NT) trials, a low-pitch double-beep signaled safety. Reappraisal (R) trials were then signaled by the word "regulieren" ("regulate"), no-reappraisal (NR) trials by the word "belassen" ("leave" or "do not regulate"). Subjects remained eyes closed throughout trials. (B) To capture the predicted dynamic network behavior during reappraisal, neural activation during trials was modeled as tonic, linearly increasing and linearly decreasing responses (see Materials and Methods). 
criterion and an important factor in determining whether a stimulus induces a lasting emotional reaction (Phillips et al., 2003; Sander et al., 2005) which is a logical pre-requisite for investigating late reappraisal activity. Third, we gave subjects detailed reappraisal instructions rather than leaving it to them how to exactly reinterpret the situation, because we reasoned that this would further reduce variance. Another motivation for this measure was to enhance chances that subjects do use reappraisal and not some other regulation technique, a problem that is pertinent in reappraisal studies where full control over the experimental manipulation is always limited by reappraisal being a purely mental process. Fourth, a final deviation from most reappraisal studies, which usually only compare a reappraisal to a no-reappraisal condition during emotional stimulation, is the use of a two by two factorial design. In such designs, one cannot only test for main effects of emotional stimulation (here, threat):

$\mathrm{T}-\mathrm{NT}=-\mathrm{NT} / \mathrm{NR}-\mathrm{NT} / \mathrm{R}+\mathrm{T} / \mathrm{NR}+\mathrm{T} / \mathrm{R}$ or $\left[\begin{array}{llll}-1 & -1 & 1 & 1\end{array}\right]$ or reappraisal:

$\mathrm{R}-\mathrm{NR}=-\mathrm{NT} / \mathrm{NR}+\mathrm{NT} / \mathrm{R}-\mathrm{T} / \mathrm{NR}+\mathrm{T} / \mathrm{R}$ or $\left[\begin{array}{llll}-1 & 1 & -1 & 1\end{array}\right]$

but also for two complementary types of interaction. The interaction contrast:

$(\mathrm{T}-\mathrm{NT})_{\mathrm{NR}}-(\mathrm{T}-\mathrm{NT})_{\mathrm{R}}=-\mathrm{NT} / \mathrm{NR}+\mathrm{NT} / \mathrm{R}+\mathrm{T} / \mathrm{NR}-\mathrm{T} / \mathrm{R}$ or $\left[\begin{array}{llll}-1 & 1 & 1 & -1\end{array}\right]$

tests for threat-related effects (T-NT) in anxiety measures or brain activity that are attenuated by reappraisal (in other words, anxiolysis). We use this contrast as a quantitative reappraisal success index (RSI) in the analysis of our behavioral data.

The inverse interaction contrast:

$(\mathrm{R}-\mathrm{NR})_{\mathrm{T}}-(\mathrm{R}-\mathrm{NR})_{\mathrm{NT}}=+\mathrm{NT} / \mathrm{NR}-\mathrm{NT} / \mathrm{R}-\mathrm{T} / \mathrm{NR}+\mathrm{T} / \mathrm{R}$ or [1 $-1-1$ 1 $]$

tests for reappraisal-related effects (R-NR), e.g., in brain activity, that are larger under threat, that is, when one has to stay or become detached in spite of a concurrent emotional challenge. Hence, both interaction contrasts can provide additional interesting information. Crossing of the reappraisal factor with the threat factor was possible here because one can detach from any kind of situation, including a less emotional one (condition NT/R).

\section{SUBJECTS}

Twenty-one right-handed healthy male subjects with an average age of $28 \pm 4$ (mean \pm SD) years (range 21-38 years) participated in the experiment. Subjects reported no current or past neurological or psychiatric illness, including anxiety disorders. All were of Caucasian origin; 15 were University students. Their average trait anxiety (Spielberger, 1985) was 32.1 \pm 4.8 (range 25-45) and thus in line with norm population values (compare Laux et al., 1981). All subjects gave informed consent. The study was approved by the ethics committee of the Hamburg Medical Board and conformed to all relevant regulatory standards. Remuneration for participation was 40 Euros.

\section{REAPPRAISAL STRATEGY AND PROCEDURE}

We chose to use a distancing reappraisal strategy by which subjects, rather than immersing themselves in the situation and experiencing potential threat and the ensuing anxiety as directly affecting them, took a detached observer position and told themselves that all on-going external and internal events were not self-relevant.
Such reappraisal has previously been shown to successfully attenuate anticipatory anxiety (Houston and Holmes, 1974; Kalisch et al., 2005). Other variants of distancing reappraisal have been used to alleviate depressed mood (Kross and Ayduk, 2008) or affective responding to negative picture material (e.g., Ochsner et al., 2004; Dillon et al., 2007; Erk et al., 2010). For the purpose of distancing, subjects were told to build a mental image of a cloud in the sky that would symbolize the current external situation such as being in the scanner, being safe from shock (NT) or being threatened ( $\mathrm{T}$ ), as well as accompanying internal sensations, feelings, and thoughts such as relaxation or relief (NT) or tension and anxiety $(\mathrm{T})$. Hence, the image of the cloud was applicable to both the No-threat and the Threat condition but might symbolize different situations/feelings/thoughts depending on the condition. They were then asked to imagine themselves far away from this cloud, for example standing on a hill and observing the cloud from a distance (but not to look away). In addition to this mental image, they were given a self-statement that expressed the detached perspective: "Die Wolke ist weit weg am Horizont. Ich betrachte sie aus der Ferne." ("The cloud is far out on the horizon. I observe it from a distance.")

On the day of the experiment, subjects were explained the strategy and then trained in using it by first having them read aloud the statement 10 times, then having them freely recall each statement 5 times and finally provide verbal ratings of the effort necessary to recall the statement (0: not effortful at all - 10: extremely effortful) and of its emotional valence ( 0 : very pleasant -10 : very unpleasant). All subjects were able to perfectly recall the statement, and final effort ratings were $1.2 \pm 1.2$. Final valence ratings were in the neutral range $(3.7 \pm 1.4)$. Subjects then had to spend 1 min eyes closed, performing visual imagery, followed by a free description of the imagined scene in their own words and ratings of effort, intensity or vividness of the images ( 0 : not vivid at all - 10: very vivid) and valence ( 0 : very negative - 10 : very positive). In every subject, the imagined scene was in agreement with the distancing strategy and could be easily produced (final effort ratings: $2.5 \pm 1.6$ ). Imagery was sufficiently vivid (intensity ratings: $6.3 \pm 1.4$ ) and neutral in valence (valence ratings: $4.6 \pm 0.5$ ).

Training was followed by pain stimulus calibration. Stimuli were applied to the back of the right hand using a Digitimer DS7A electrical stimulator (Digitimer Ltd., Welwyn Garden City, UK) delivering $2 \mathrm{~ms}$ square-wave pulses of 0.01-100 mA through a surface electrode with platinum pin (Clyde's Polo Kit Supplies, Bexley, UK). Current levels were chosen which induced intermediate subjective anxiety. To achieve this, subjects rated their anxiety during a 15-to- 0 countdown on a 100 point-scale. Subjects were told they might receive a triple-stimulus of a previously experienced level at any time during the countdown at a probability of $25 \%$. This procedure was repeated with different current levels, starting at low levels, until an anxiety level between 50 and 80 was reached.

In a brief pre-experimental session, subjects were then familiarized with the experiment inside the scanner, but without receiving any pain stimulus. The actual experiment (see below) was split into three functional runs of 13 min duration each. Before each run, the pain stimulus was tested (rated) and recalibrated if necessary. This was done to preclude reinforcer devaluation effects that might 
otherwise explain the anxiolytic effects of reappraisal. In addition, after each run subjects provided the same ratings on statement and imagery as after training (see above). Subjects' efforts to subvocally rehearse the statement and to mentally imagine the scene were slightly higher during scanning than at training but stable across runs (statement: $2.9 \pm 1.5,2.9 \pm 1.4$, and $3.1 \pm 1.3$; scene: $3.9 \pm 2,3.9 \pm 1.6$, and $4.2 \pm 2$ ). The vividness of imagery was comparatively reduced but also stable $(5.9 \pm 1.8,5.7 \pm 1.9$, and $5.9 \pm 2.2$ ). The emotional valence of the statement and the imagined scene was stably judged as neutral to mildly pleasant (statement: $3.3 \pm 1.5,3.1 \pm 1.5$, and $3.5 \pm 1.5$; scene: $3.9 \pm 1.3$, $3.8 \pm 1.3$, and $4.6 \pm 1.9$ ). The latter is important to exclude that emotion regulation in this task consisted in simply replacing negative affect by some strong, opposing positive emotion (that is, self-distraction). After the experiment, subjects were interviewed about the strategies they used and about their experiences during the scans. No subject had to be excluded due to apparent unsatisfactory commitment.

\section{TASK}

There were altogether 84 randomized $17.7 \mathrm{~s}$ trials (28 per run), 18 in each of the conditions NT/NR and NT/R and 24 in each of the conditions T/NR and T/R (see Figure 1 for trial structure and cues). During six of the T/NR and T/R trials subjects received a triple pain stimulus (pulse intervals: $80 \mathrm{~ms}$ ) which occurred randomly within a time window of 3-12 s after offset of the auditory instruction (that is, from approx. $2.7 \mathrm{~s}$ into the trial). A trial ended with the instruction "Augen auf, Rating!" ("Eyes open, rating.") followed by a 5-s presentation of a rating screen with the question "Wie groß war Ihre Angst/Anspannung?" ("How strong was your anxiety/tension?") and a visual analog scale below. On the scale, subjects could move a red star using their keypad between poles "no anxiety" (0) and "very strong anxiety" (100). The position of the star at the onset of each rating was randomized. The subsequent $5 \mathrm{~s}$ break was cued by the words "Augen zu, Pause!" ("Eyes closed, break.").

\section{DATA ACOUISITION}

Skin conductance (SC) was measured at a sampling rate of $1000 \mathrm{~Hz}$ from electrodes on the palm and the thenar of the left hand using a CED2502-SA SC unit (Cambridge Electronic Design, Cambridge, UK) and recorded with Spike 2 software (CED). Offline, SC data were down-sampled to $100 \mathrm{~Hz}$ and filtered (Gaussian kernel, FWHM $0.1 \mathrm{~s}$ ). SC level (SCL) was defined as the average SC across a trial minus SC at the first time point in that trial. Values were $z$-transformed (Buchel et al., 1998). In one subject, SC data could not be used due to technical problems, reducing sample size for SC analysis to $n=20$.

Functional imaging was performed on a 3-Tesla MR scanner (Siemens Trio, Erlangen, Germany) equipped with a 12channel head coil, using a gradient echo $\mathrm{T} 2{ }^{*}$ weighted echo-planar imaging (EPI) sequence with blood oxygenation level-dependent $(\mathrm{BOLD})$ contrast $\left(\mathrm{TE}=30 \mathrm{~ms}, \mathrm{TR}=2.47 \mathrm{~s}\right.$, flip angle $\left.=80^{\circ}\right) . \mathrm{TE}$ was minimized using a parallel acquisition technique (generalized autocalibrating partially parallel acquisitions, GRAPPA) with an acceleration factor of 2 and 24 reference lines. Each volume comprised 38 axial slices (AC-PC orientation) of $2 \mathrm{~mm}$ thickness and $2 \mathrm{~mm} \times 2 \mathrm{~mm}$ in-plane resolution with a slice gap of $1 \mathrm{~mm}$. Participants were placed in a light head restraint within the scanner to limit head movement during acquisition. A T1-weighted structural image was also acquired.

\section{DATA ANALYSIS}

Trials during which subjects received pain stimuli were excluded from all analyses of experimental effects. Statistical analysis of behavioral data was performed within SPSS 17.0 (SPSS, Chicago, IL, USA).

Functional magnetic resonance imaging (fMRI) data were preprocessed using SPM8 (www.fil.ion.ucl.ac.uk/spm; Friston et al., 2007). The five initial EPI images were discarded to account for T1 equilibration. To correct for head movement and movementby-distortion interactions, they were then realigned to the sixth volume and unwarped. The structural $\mathrm{T} 1$ images were coregistered to the EPI images and then segmented and spatially normalized to a standard T1 template using the "New Segment"-routine as implemented in SPM8. The normalization parameters from this procedure were then applied to the EPI images. The normalized EPI images were spatially smoothed (Gaussian kernel, FWHM $6 \mathrm{~mm}$ ), temporally high-pass filtered (cut-off $128 \mathrm{~s}$ ) and corrected for temporal autocorrelations using first-order autoregressive modeling.

Statistical analysis was performed using a standard approach for fMRI, involving a general linear convolution model at the single-subject level and a random-effects analysis at the group level within the SPM software (see Friston et al., 2007, for details). The three runs were concatenated into a single time series and, for each subject, regressors were defined that modeled the predicted time courses of experimentally induced brain activation changes. Each of the four experimental conditions (NT/NR, NT/R, T/NR, T/R) was modeled using two different temporal response profiles during the 17.7-s trials: a tonic response lasting the whole duration of a trial and a response that increased linearly across a trial (see Figure 1). Receipt of pain was modeled as distinct "events" (delta functions with 0 duration). Blocks during which subjects actually received pain stimuli and ratings were modeled as "boxcar" (on-off) regressors. Onset of pauses after the ratings were modeled as events. Each regressor was convolved with the canonical hemodynamic response function. Using these regressors in a general linear model (multiple regression) of brain activation at each voxel yields parameter estimates of the contribution of each regressor to the fMRI signal measured in each voxel. The subject- and regressor-specific parameter estimate images were spatially smoothed (FWHM $10 \mathrm{~mm}$ ) and, for the standard analysis reported in Results, entered into a random-effects group analysis using SPM's "flexible factorial” model which permits correction for possible non-sphericity of the error term (here, dependence of conditions). Group-level design matrices included 25 regressors ( 4 regressors of interest corresponding to the 4 experimental conditions NT/NR, NT/R, T/NR, and T/R, plus 21 subject constants). Linear combinations ("contrasts") of the regressors of interest were used to test for main effects and interactions as defined in the Overview section above. Here, multiplication of the parameter estimate images for the linearly increasing regressors by -1 allowed for also assessing linearly decreasing responses 
as depicted in Figure 1. Significance of effects was tested using voxel-wise one-tailed $t$-tests.

For the standard analysis, correction for multiple comparisons following Gaussian random field theory (family wise error, FWE method) at a threshold of $p<0.05$ ("small volume correction") was limited to regions of interest (ROIs) for which we had a priori hypotheses and which we identified from the literature. Specifically, for main effects and interaction contrasts related to threat responding we used the two activation peaks which had shown maximally consistent activation across studies in a meta-analysis of instructed fear experiments (Mechias et al., 2010), one located in the dorsomedial prefrontal/anterior cingulate cortex (dmPF/ACC, MNI coordinates $x, y, z=0,16,36)$ and one in the right anterior insula $(36,20,0)$. Like in previous work (Raczka et al., 2010), the dmPFC/ACC ROI was a box of dimensions $x, y, z=20,16,16 \mathrm{~mm}$, covering the mPFC bilaterally. The insula ROI was a sphere with a $12-\mathrm{mm}$ radius. Note that the instructed fear paradigm does not reliably and consistently activate the amygdala (see Mechias et al., 2010, for meta-analysis) and does not do so in our hands, even when taking into account possible habituation (Kalisch et al., 2005). Recent developments have highlighted a role for the $\mathrm{dmPFC} / \mathrm{ACC}$ as another important mediator of fear expression in both animals and humans (reviewed in Etkin et al., 2011). For main effects and interaction contrasts related to reappraisal we used the two activation peaks which had shown maximally consistent activation across studies in a meta-analysis of reappraisal experiments (Kalisch, 2009), one in the dmPFC $(-4,20,52)$ and one in the left LFC $(-40,10,48)$. The corresponding box in the dmPFC was midlinecentered $(x=0)$ to cover the dmPFC bilaterally. The LFC ROI was again a sphere with a $12-\mathrm{mm}$ radius. In addition, we used the peak effect from our previous detachment-from-threat study (Kalisch et al., 2005) where we had seen increasing reappraisal activity over episodes in a right anterior LFC focus $(42,48,18$; 12-mm sphere).

In the results tables, anatomical localization of activations was carried out with reference to the atlas of Duvernoy (1999). Unambiguous white matter or liquor clusters are not reported. Cluster submaxima are reported when more than $8 \mathrm{~mm}$ apart.

For the test of IMMO by comparison of the spatial distributions of linearly increasing and decreasing reappraisal main effects (see Results), we created an anatomical mask of the bilateral LFC that included all parts of the superior, middle, and inferior frontal gyri, based on a standard anatomical atlas (Tzourio-Mazoyer et al., 2002).

\section{RESULTS}

BEHAVIORAL RESULTS: ANXIETY RATINGS AND SKIN CONDUCTANCE

Anxiety ratings showed a significant main effect of Threat $[F(1,20)=367.37, p<0.001]$, a trend-like main effect of Reappraisal $[F(1,20)=4.11, p=0.056]$, and a significant Threat by Reappraisal interaction $[F(1,20)=10.17, p=0.005]$. The interaction was apparently driven by a reduction of anxiety in the T/R compared to the T/NR condition (Figure 2A), an anxiolytic effect corresponding to the interaction term [ $\left.-\begin{array}{llll}-1 & 1 & 1 & -1\end{array}\right]$ or RSI as defined above in "Materials and Methods," Overview section. The anxiety-rating-RSI was significantly $>0[9.1 \pm 13, t(20)=3.19$, $p=0.003$ one-tailed, planned post hoc $t$-test]. A caveat is that, rather than a true reduction of anxiety by reappraisal, these ratings may also reflect the demand characteristics of the task. SC is an index of the sympathetic arousal that usually accompanies anxiety and thus a more objective metric for anxiety. SC was significantly elevated by threat [main effect of threat: $F(1,19)=97.4$, $p<0.001$ ], not affected by reappraisal as such [main effect of reappraisal: $F(1,19)=0.26, p=0.619]$, and showed the critical interaction of Threat and Reappraisal $[F(1,19)=5.37, p=0.032$;

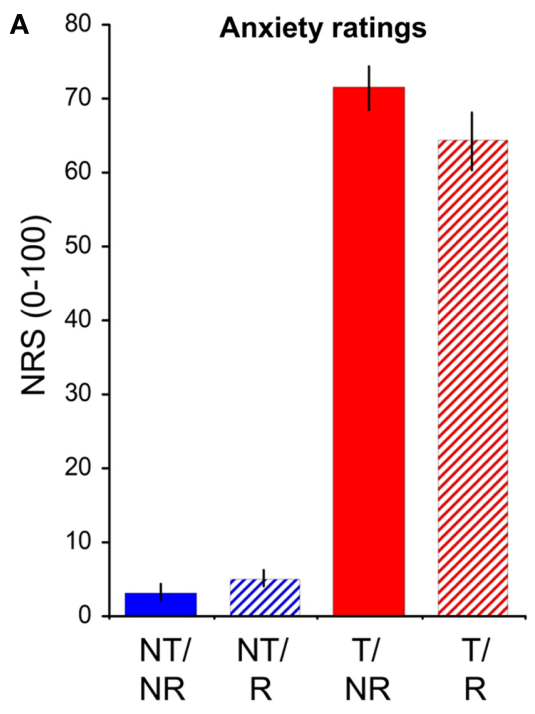

FIGURE 2 | Behavioral results. Average trial-by-trial anxiety ratings (A) and skin conductance levels (B) in the conditions no-threat/no-reappraisal (NT/NR),

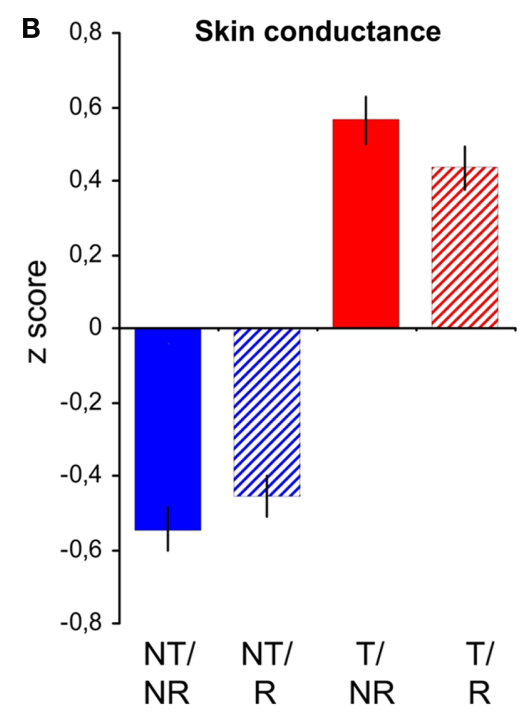

no-threat/reappraisal (NT/R), threat/no-reappraisal (T/NR), and threat/reappraisal (T/R). Error bars: SEM. NRS, numerical rating scale. 
Figure 2B $]$. The SC-RSI was significant $[0.22 \pm 0.42, t(19)=2.32$, $p=0.016$ one-tailed], further confirming the anxiolytic effect of reappraisal.

\section{IMAGING RESULTS: STANDARD ANALYSIS}

For an overview, main effects and interactions in tonic and linearly increasing and decreasing responses are reported in Tables 1-3. Globally, threat of shock induced the typical widespread activations in dmPFC/dACC, anterior insula, basal ganglia, thalamus, brainstem, cerebellum, and other areas (compare Mechias et al., 2010, for meta-analysis). In our predefined $\mathrm{dmPFC} / \mathrm{dACC}$ and right anterior insula ROIs (see Materials and Methods), there were significant tonic (dACC/dmPFC: 2,20,34, $z$ score $=3.63, p=0.004$ corrected for multiple comparisons; right anterior insula: $34,26,4, z=5.13, p<0.001$ corr., and others) and decreasing (dmPFC/dACC: $-2,10,36, z=2.92, p=0.028$ corr.; right anterior insula: $38,26,4, z=3.23, p=0.013$ corr.) main effects of threat.

Main effects of reappraisal were observed in the previously described network comprising medial and lateral frontal areas, parietal, and temporal cortex, cerebellum, basal ganglia, and others (compare Kalisch, 2009, for meta-analysis). In our predefined dmPFC, left LFC and right anterior LFC ROIs (see Materials and Methods), there were significant tonic (dmPFC: $-8,12,48, z=5.87, p<0.001$ corr.; left LFC: $-46,0,46, z=7.45$, $p<0.001$ corr.; Figures 3A,B), increasing (dmPFC: $-2,28,48$, $\mathrm{z}=2.91, p=0.029$ corr.; left LFC: $-40,20,42, \mathrm{z}=3.11, p=0.019$ corr.; right anterior LFC: $42,44,8, \mathrm{z}=3.25, p=0.012$ corr.; Figures 3C-E), and decreasing (left LFC: $-44,0,44, z=3.45$, $p=0.007$ corr.; Figure $3 \mathrm{~F}$ ) responses. The parameter estimates in the figure suggest that some of these responses tended to be higher when subjects performed reappraisal under threat (condition T/R) compared to when they were safe (condition NT/R). This apparent threat by reappraisal interaction however rarely reached significance (see below), presumably because it was masked by the massive reappraisal main effect.

Threat by reappraisal interactions of the form $\left[\begin{array}{llll}-1 & 1 & 1 & -1\end{array}\right]$, corresponding to threat-related activity that is reduced by reappraisal (see Materials and Methods, Overview section), were sparse (see Tables 1-3). The only significant effect was found in our right anterior insula ROI for linearly decreasing responses $(36,28,4, z=2.97$, $p=0.027$ corr., and others; Figure 4A), in an area close to the peak showing a decreasing main effect of threat (see above and Table 3 ). The pattern of parameter estimates in Figure 4A shows that reappraisal (condition $\mathrm{T} / \mathrm{R}$ ) abolished the signal decrease otherwise observed as a response to threat (condition T/NR), in line with an anxiolytic effect of reappraisal.

Inverse threat by reappraisal interactions of the form $[1-1$ $-11]$, corresponding to reappraisal-related activity that is larger under threat (see Materials and Methods, Overview section), were mainly observed outside the frontal cortex (Tables 1-3). A trendlevel effect was found in our dmPFC ROI for linearly increasing responses $(6,26,60, z=2.44, p=0.086$ corr.; Figure $4 \mathbf{B})$.

Globally speaking, these results are in agreement with previous imaging studies and further confirm the behavioral findings of successful anxiety induction by threat of shock and successful down-regulation of anxiety (anxiolysis) by detachmentreappraisal.

\section{IMAGING RESULTS: TESTING IMMO}

If early and late stages of reappraisal rely on different neural substrates, then reappraisal-related activations with linearly decreasing and increasing response profiles should show dissociable anatomical distributions. More specifically, if the observations in Kalisch (2009) hold, then linearly decreasing effects should be mainly observed in the left posterior LFC while increasing effects should be mainly located in the right anterior LFC. The SPM glass brains for both types of reappraisal main effect contrasts in Figures 5A,B seem to confirm this prediction, with exclusive left posterior activation in the decreasing contrast and additional and more prominent recruitment of right-sided areas in the increasing contrast.

To formally test this, we used methodology analogous to our previous meta-analysis (Kalisch, 2009). We averaged within each of the 21 subjects in the study and for each type of contrast (decreasing, increasing reappraisal main effect) the coordinates of all (leftand right-sided) activated voxels contained in the a priori bilateral LFC mask defined in Section "Materials and Methods," at an uncorrected threshold of $p<0.01$. This threshold turned out to be most appropriate as it yielded supra-threshold voxels in nearly all subjects (20 for increasing responses, 19 for decreasing responses), thus assuring a sufficient number of data points and comparability across response types. Averaging of coordinates resulted in one single coordinate for each subject and contrast (Figure 6) that expressed the "center of gravity" of lateral frontal reappraisal activation in that subject and contrast. That is, if in a given subject and contrast the majority of activated voxels was located in, for instance, the left LFC, this would result in an average coordinate with a negative (left-sided) $x$ value. Analogously, if in a given subject and contrast the majority of LFC voxels was located in, for instance, posterior LFC, this would "push" the average coordinate toward smaller (more posterior) $y$ values. Note this "center of gravity" is a virtual coordinate which may not correspond to any actual locus of activation. For the decreasing response, the group average of these coordinates was $x, y, z=-38,13,33$ and thus located significantly more to the left (smaller $x$ value) and more posteriorly (smaller $y$ value) than the group-averaged coordinate for the increasing response $[x, y, z=12,36,19 ; x$ value: $t(17)=4.76$, $p<0.001 ; y$ value: $t(17)=2.91, p=0.005$; one-tailed paired twosample $t$-tests]. This result was robust to removal of outliers $( \pm 2 \mathrm{SD}$, all $p<0.003)$.

In addition, we observed an unpredicted effect in the $z$ values $[t(17)=2.39, p=0.029$ two-tailed; after removal of outlier: $p=0.019$; not shown], with decreasing responses being located slightly more dorsally (higher $z$ value). A further unpredicted finding was that the tonic reappraisal main effect showed an average center of gravity $(x, y, z=-12,7,17)$ that had a similar left posterior location as the decreasing response (Figures $\mathbf{5}$ and $\mathbf{6}$ ).

\section{DISCUSSION}

Our data provide first evidence for the existence of at least two separable stages of mental activity occurring during a sufficiently long reappraisal episode, one early stage apparently marked by left 
Table 1 | Imaging results: standard analysis. Activations with tonic response profile across trials.

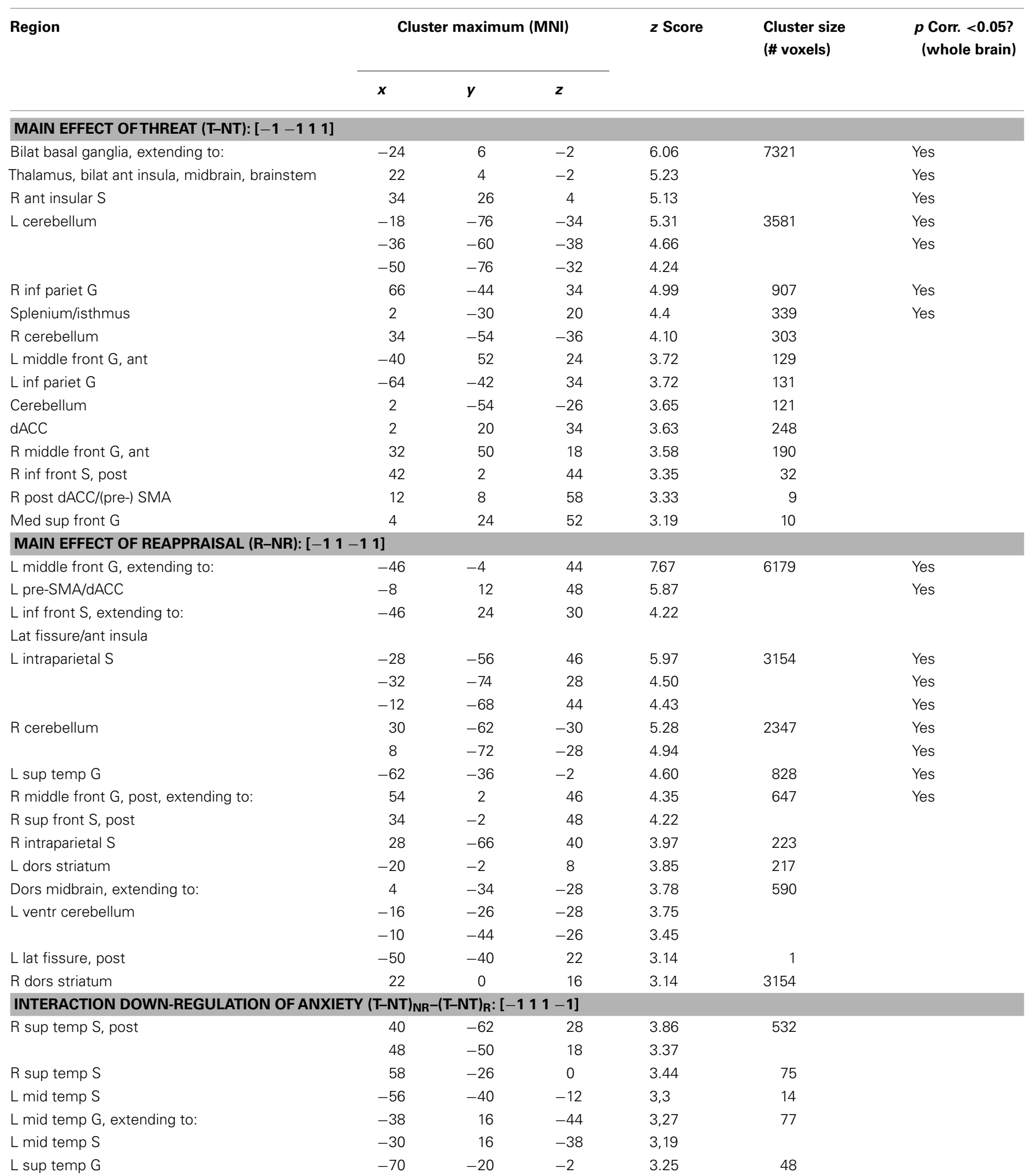

INTERACTION THREAT-SPECIFIC REAPPRAISAL (R-NR) - (R-NR) $)_{\mathrm{NT}}$ : [1 - 1 - 1 1]

No voxels surviving threshold

Statistical threshold: $p<0.001$ uncorrected. Definition of contrast as in Section "Materials and Methods," Overview section. G, gyrus; L, left; R, right; S, sulcus. MNI, Montreal Neurological Institute. 
Table 2 | Imaging results: standard analysis. Activations with linearly increasing response profile across trials.

\begin{tabular}{|c|c|c|c|c|c|c|}
\hline \multirow[t]{2}{*}{ Region } & \multicolumn{3}{|c|}{ Cluster maximum (MNI) } & \multirow[t]{2}{*}{ z Score } & \multirow{2}{*}{$\begin{array}{l}\text { Cluster size } \\
\text { (\# voxels) }\end{array}$} & \multirow{2}{*}{$\begin{array}{l}p \text { Corr. }<0.05 ? \\
\text { (whole brain) }\end{array}$} \\
\hline & $\boldsymbol{x}$ & $y$ & $\boldsymbol{z}$ & & & \\
\hline \multicolumn{7}{|c|}{ MAIN EFFECT OFTHREAT (T-NT): [-1 - 1111$]$} \\
\hline L ant dors striatum & -18 & 14 & 8 & 3.17 & 7 & \\
\hline L post caudatum/white matter & -24 & -26 & 22 & 3.15 & 5 & \\
\hline \multicolumn{7}{|c|}{ MAIN EFFECT OF REAPPRAISAL (R-NR): [-1 $1-1$ 1] } \\
\hline R temp-parietal-occipital transition & 58 & -38 & 36 & 4.69 & 2372 & Yes \\
\hline zone, incl. intraparietal S & 54 & -46 & 34 & 4.58 & & Yes \\
\hline $\mathrm{R}$ middle temp $\mathrm{G}$ & 72 & -16 & -20 & 4.15 & 228 & \\
\hline$R$ sup front $\mathrm{S}$ & 38 & 14 & 42 & 3.89 & 865 & \\
\hline$L$ inf pariet $G$ & -56 & -52 & 38 & 3.89 & 644 & \\
\hline R insular G, post & 36 & -2 & 2 & 3.86 & 129 & \\
\hline L lat OFC & -46 & 50 & -10 & 3.85 & 251 & \\
\hline$L$ inf temp $G$ & -52 & -2 & -36 & 3.66 & 167 & \\
\hline R post orbit $G$ & 36 & 40 & -14 & 3.62 & 60 & \\
\hline$R$ sup front $S$ & 20 & 40 & 32 & 3.6 & 163 & \\
\hline$L$ middle temp $\mathrm{G}$ & -64 & -30 & -18 & 3.57 & 311 & \\
\hline R insular S, vent & 30 & 18 & -20 & 3.38 & 73 & \\
\hline$R$ inf front $S$, ant & 40 & 44 & 4 & 3.36 & 130 & \\
\hline L cerebellum & -30 & -70 & -38 & 3.28 & 20 & \\
\hline $\mathrm{L}$ ant dACC/pgACC & -6 & 46 & 16 & 3.28 & 146 & \\
\hline$L$ middle front $S$ & -42 & 22 & 42 & 3.13 & 5 & \\
\hline \multicolumn{7}{|c|}{ INTERACTION DOWN-REGULATION OF ANXIETY $(T-N T)_{N R}-(T-N T)_{R}:\left[\begin{array}{llll}-1 & 1 & -1\end{array}\right]$} \\
\hline L supramarginal G & -38 & -50 & 32 & 3.65 & 106 & \\
\hline L cerebellum & -56 & -62 & -38 & 3.38 & 54 & \\
\hline \multirow[t]{2}{*}{ L cerebellum } & -2 & -92 & -30 & 3.34 & 96 & \\
\hline & -8 & -82 & -28 & 3.18 & & \\
\hline L cerebellum & -50 & -78 & -28 & 3.32 & 30 & \\
\hline L cerebellum & -40 & -60 & -28 & 3.17 & 9 & \\
\hline
\end{tabular}

INTERACTIONTHREAT-SPECIFIC REAPPRAISAL (R-NR) $-(R-N R)_{\mathrm{NT}}:\left[\begin{array}{lll}1-1 & -1 & 1\end{array}\right]$

No voxels surviving threshold

Statistical threshold: $p<0.001$ uncorrected. Definition of contrast as in Section "Materials and Methods," Overview section. G, gyrus; L, left; OFC, orbitofrontal cortex; pgACC, perigenual ACC; R, right; S, sulcus; SMA, supplementary motor area. MNI, Montreal Neurological Institute.

Table 3 | Imaging results: standard analysis. Activations with linearly decreasing response profile across trials.

\begin{tabular}{|c|c|c|c|c|c|c|}
\hline \multirow[t]{2}{*}{ Region } & \multicolumn{3}{|c|}{ Cluster maximum (MNI) } & \multirow[t]{2}{*}{ z Score } & \multirow{2}{*}{$\begin{array}{l}\text { Cluster size } \\
\text { (\# voxels) }\end{array}$} & \multirow{2}{*}{$\begin{array}{l}p \text { Corr. }<0.05 ? \\
\text { (whole brain) }\end{array}$} \\
\hline & $\boldsymbol{x}$ & $y$ & $z$ & & & \\
\hline \multicolumn{7}{|c|}{ MAIN EFFECT OF THREAT (T-NT): [-1 $\left.-1 \begin{array}{lll}-1 & 1\end{array}\right]$} \\
\hline L sup pariet $\mathrm{G}$, med & -4 & -62 & 72 & 4.08 & 247 & \\
\hline R cuneus & 8 & -104 & 8 & 3.29 & 35 & \\
\hline $\mathrm{R}$ insular $\mathrm{S}$, ant & 38 & 26 & 4 & 3.24 & 19 & \\
\hline \multicolumn{7}{|c|}{ MAIN EFFECT OF REAPPRAISAL (R-NR): [-1 $1-1$ 1] } \\
\hline L sup front $S$ & -44 & -2 & 44 & 3.7 & 103 & \\
\hline L sup front G, med/(pre-) SMA & -6 & 2 & 66 & 3.58 & 68 & \\
\hline \multicolumn{7}{|c|}{ INTERACTION DOWN-REGULATION OF ANXIETY (T-NT) $)_{N R}-(T-N T)_{R}:\left[\begin{array}{llll}-1 & 1 & 1 & -1\end{array}\right]$} \\
\hline \multicolumn{7}{|c|}{ See interaction threat-specific reappraisal $(R-N R)_{T}-(R-N R)_{N T}:\left[\begin{array}{llll}1 & -1 & -1 & 1\end{array}\right]$ in Table 2} \\
\hline \multicolumn{7}{|c|}{ INTERACTION THREAT-SPECIFIC REAPPRAISAL (R-NR) $-(R-N R)_{\mathrm{NT}}:[1-1-11]$} \\
\hline see Interaction Down-regulatio & ty $(\mathrm{T}-\mathrm{N}$ & $(\mathrm{VT})_{\mathrm{R}}:[-$ & in $\mathrm{Ta}$ & & & \\
\hline
\end{tabular}

Statistical threshold: $p<0.001$ uncorrected. Definition of contrast as in Section "Materials and Methods," Overview section. G, gyrus; L, left; R, right; S, sulcus; SMA, supplementary motor area. MNI, Montreal Neurological Institute. 

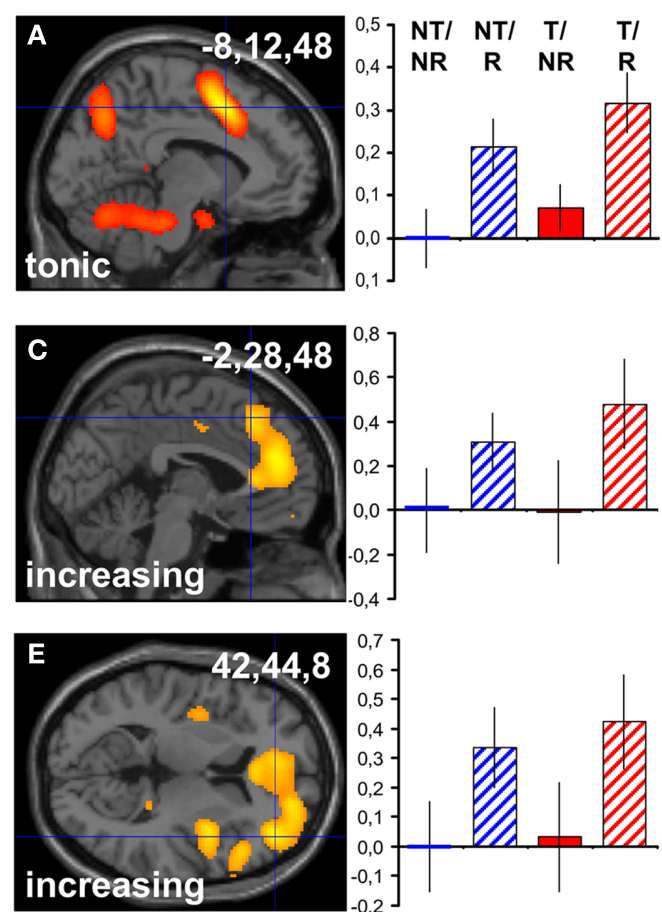

FIGURE 3 | Standard analysis: reappraisal main effects. Reappraisa main effects (contrast R-NR or [ $\left.\begin{array}{llll}-1 & 1 & -1 & 1\end{array}\right]$, see Materials and Methods, overview section) in predefined ROls surviving small volume correction for multiple comparisons at $p<0.05$. Tonic response in $\mathrm{dmPFC}$ (A) and left LFC (B). Linearly increasing response in dmPFC (C), left LFC (D), and right anterior LFC (E). Linearly decreasing response in left LFC (F). The

Reappraisal main effects with a linearly decreasing profile are computed after multiplying single-subject parameter estimate images for the linearly increasing regressor with -1 (see also Materials and Methods, Data
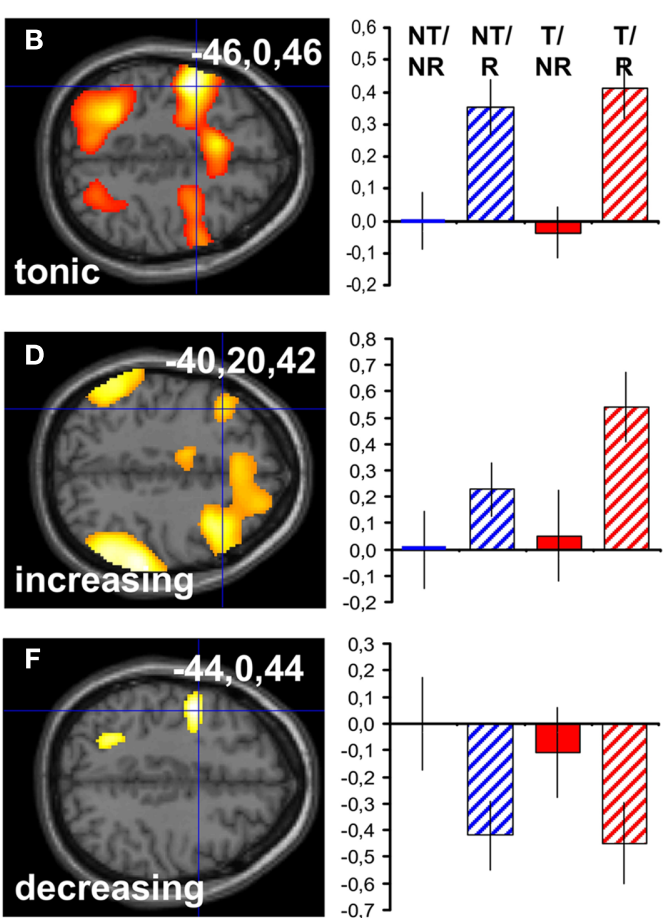

analysis). Activations are superimposed on a canonical structural image. Display threshold: $p<0.01$ uncorrected. Left is upward in the coronal views. Bar graphs show corresponding group-averaged parameter estimates ("betas") in the voxel indicated by the haircross and the inserted coordinate. Values are normed to the first condition (NT/NR). In tonic contrasts, positive parameter estimates indicate tonic activation. In the other contrasts, positive parameter estimates indicate linearly increasing, negative parameter estimates indicate linearly decreasing response profiles. Error bars: SEM.
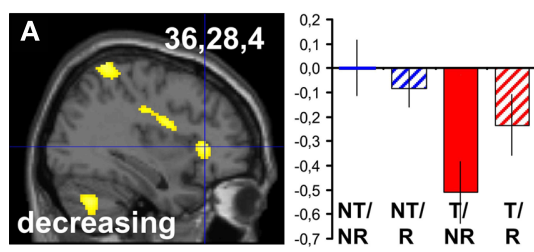

FIGURE 4 | Standard analysis: threat by reappraisal interactions. Threat by reappraisal interactions in predefined $\mathrm{RO}$ Is surviving small volume correction for multiple comparisons at $p<0.05$ (A) or at trend-level, $p<0.1$ (B). In the right anterior insula (A), a linearly decreasing response to threat (condition T/NR) is attenuated by reappraisal (condition T/R). The peak was identified from the interaction contrast [ $\left[\begin{array}{llll}-1 & 1 & 1 & -1\end{array}\right]$ after multiplying the single-subject parameter estimate images for the linearly increasing regressor with -1 (see Materials and Methods). In the dmPFC (B),

reappraisal induces a linearly increasing response specifically when subjects

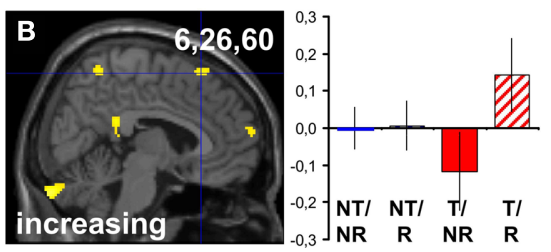

are threatened (condition T/R) but not when they are safe (condition NT/R) The peak was identified from the interaction contrast [1 - $1-1$ 1] (see Materials and Methods). Activations are superimposed on a canonical structural image. Display threshold: $p<0.01$ uncorrected. Bar graphs show corresponding group-averaged parameter estimates ("betas") in the voxel indicated by the haircross and the inserted coordinate. Values are normed to the first condition (NT/NR). Positive parameter estimates indicate linearly increasing, negative parameter estimate indicate linearly decreasing responses. Error bars: SEM. posterior LFC activation and a later stage apparently marked by comparatively more right-sided and more anterior LFC activation. Our data do not provide evidence as to what types of cognitive processes operate during these two stages. We can therefore only assume that early processes subserve the implementation of the reappraisal strategy while late processes subserve its maintenance.
Because subjects were instructed to use a specific, well-specified strategy and had received some training before the experiment, strategy selection mechanisms are unlikely to have played a major role during the putative implementation stage. This leaves memory retrieval as a likely candidate for the left posterior LFC processes active at that stage. Possible candidates for the later stage of 
A
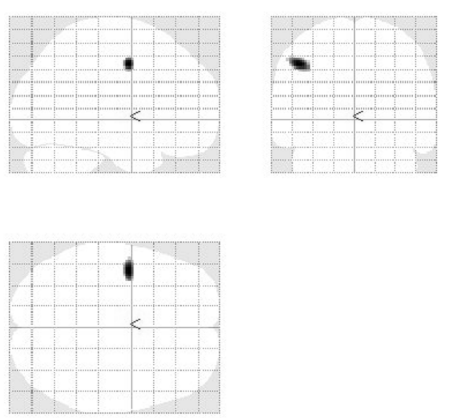

D
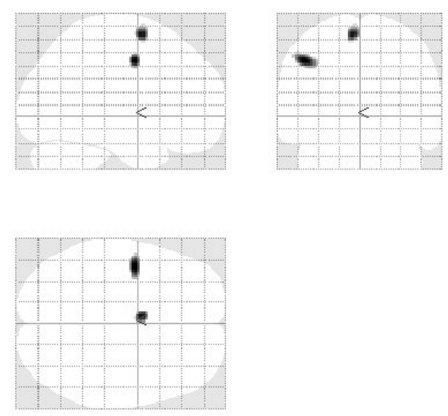

decrease
B
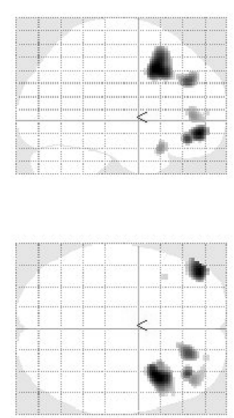

$\mathbf{E}$
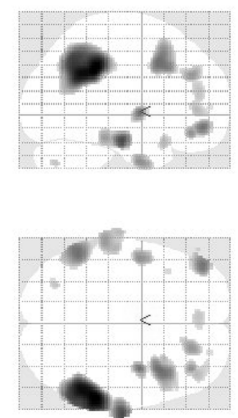

increase c
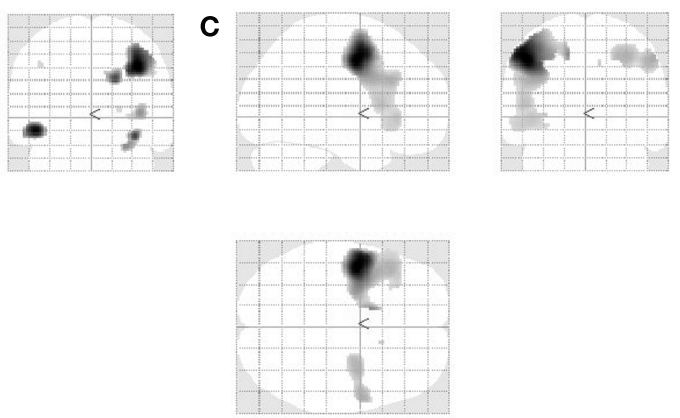

$\mathbf{F}$
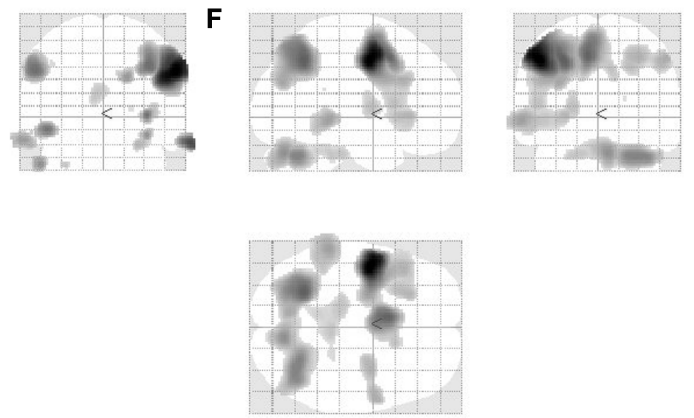

tonic
FIGURE 5 | Testing IMMO: glass brains. Reappraisal main effects (R-NR or $\left.\left[\begin{array}{llll}-1 & 1 & -1 & 1\end{array}\right]\right)$ with linearly decreasing (A-D), increasing (B-E), and tonic response profiles (C-F). Glass brains in the upper row are masked by a bilateral LFC mask (see Materials and Methods). Glass brains in the lower row additionally show extra-LFC voxels and are otherwise identical. Threshold: $p<0.001$ uncorrected. Left is left and right is right. Note the apparent shift from comparatively more left posterior to more right anterior activation sites between linearly decreasing and increasing profiles.

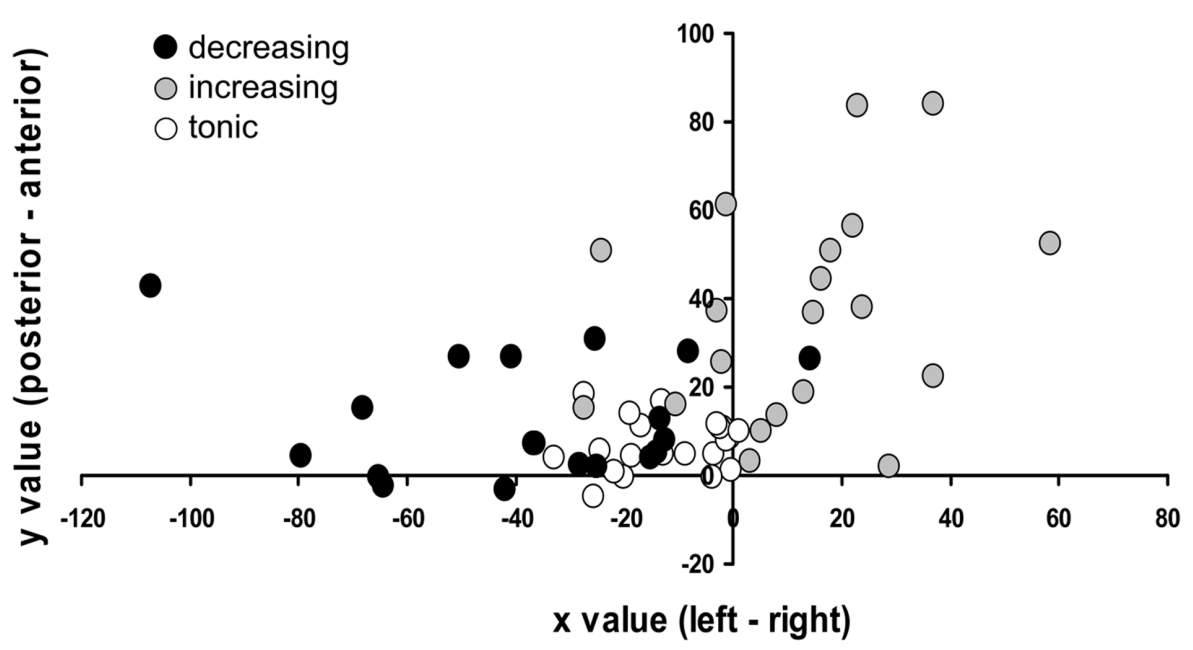

FIGURE 6 | Testing IMMO: lateral frontal average coordinates. In each subject, coordinates of all lateral frontal supra-threshold $(p<0.01$ uncorrected) voxels from the reappraisal main effect (R-NR or [ $\left.\left.\begin{array}{llll}-1 & 1 & -1 & 1\end{array}\right]\right)$ were averaged into one single, virtual coordinate or "center of gravity."
Reappraisal effects with a linearly decreasing response profile (black dots) are located comparatively more to the left ( $x$ value of average coordinate) and more posteriorly ( $y$ value) than reappraisal effects with a linearly increasing profile (gray dots). White dots: tonic profile. maintenance are working memory and performance monitoring operations.

An important question for future research will be to what extent these findings can be generalized to other types of emotions, including positive ones, and other types of reappraisal strategies, such as those that reinterpret the causal structure of a situation ("situation-focused") rather than applying distancing ("selffocused"; Ochsner et al., 2004). Within the narrower context of the 
paradigm used here, one can ask if the results would hold with a different No-reappraisal (NR) comparison condition. The issue of the comparison condition is critical because a low-level "attending to the emotion" condition like the one used here and in most other paradigms does not control for possible non-specific effects of general cognitive effort, mental imagery, linguistic processing (here: subvocal rehearsal), outcome expectation, and others. In an earlier study with a similar, though more complicated detachmentreappraisal strategy we had used a comparison condition that also included imagery and subvocal rehearsal, in order to more closely match R and NR conditions (Kalisch et al., 2005). The comparison condition had promoted immersion into the situation (compare Kross and Ayduk, 2008) in an attempt to model a natural and spontaneous appraisal of threat situations. One can predict that using such a control condition would abolish most reappraisal (R-NR) activations observed in this study, at least as far as these reflect the general executive processes needed to retrieve and maintain selfstatement and imagery. Conversely, such a comparison should be more specific for performance monitoring operations. This prediction is based on the reasoning that subjects do not intend to down-regulate their anxiety in the control condition and do not expect it to attenuate their anxiety, making success monitoring in the control condition irrelevant and superfluous. IMMO conjectures performance monitoring to normally occur during the later stages of reappraisal trials, and we have speculated that the right anterior LFC might be specifically involved in this function (Kalisch, 2009).

Further factors can be predicted to influence activation patterns. In a situation where reappraisal demands vary from trial to trial (e.g., because each emotion-inducing stimulus, such as an affective picture, varies from preceding ones in content and intensity) and subjects thus have to select slightly different reappraisals in every trial, there should be an emphasis on implementation functions, and thus comparatively more left posterior LFC activity. This should be especially true when situation-focused strategies are used (see above) which often require individual solutions for every single emotional situation and compared to which self-focused reappraisal (that is, detachment) is a more generic, all-purpose type of strategy. Implementation operations should also be comparatively more dominant when subjects have not received prior training and/or are free to choose their own preferred reappraisal strategy. The same should apply to paradigms where subjects are only cued to begin to reappraise after the onset of the emotional stimulation. Finally, we have emphasized that a simple early/late distinction is only observable if reappraisal episodes are long enough (otherwise there is no time for maintenance) and if subjects are at least moderately successful in their reappraisal efforts (otherwise there will be frequent switching between performance monitoring and enhanced implementation and/or maintenance efforts and activation patterns should become largely unpredictable; Kalisch, 2009).

Our study is limited in that it cannot address this host of questions. Yet we believe it is an important first step toward better understanding the cognitive and neural architecture of reappraisal. A further limitation that needs to be mentioned is the use of an exclusively male, Caucasian sample that, moreover, comprised mainly university students. Male and female subjects recruit similar neural networks when reappraising, but to a different degree (McRae et al., 2008; Mak et al., 2009; Domes et al., 2010). While restricting this study to male subjects should have helped in reducing variance and thus identifying potentially subtle spatiotemporal activation effects, this measure clearly necessitates replication of findings in a more representative sample. Finally, it would be desirable to reproduce our fMRI findings with other imaging modalities such as EEG or MEG (e.g., Hajcak and Nieuwenhuis, 2006; Moser et al., 2006, 2009; Deveney and Pizzagalli, 2008). It should also be mentioned that other reappraisal studies have occasionally observed lateralized activation patterns in some contrasts (e.g., Ochsner et al., 2002, 2004; Phan et al., 2005; Urry et al., 2006; Eippert et al., 2007; Kim and Hamann, 2007; Walter et al., 2009; Erk et al., 2010), although these studies did not take into account time as a factor and generally did not calculate formal laterality analyses. We have earlier emphasized the possibility that other factors than time may affect the location of reappraisal-related frontal activations (Kalisch, 2009). We are however currently not aware of any single factor that is systematically associated with either predominantly left- or right-sided activation.

In the remaining paragraphs, we would like to discuss a number of side findings that might nevertheless be interesting for future theorizing. In our previous detachment-from-threat study with an immersion comparison condition (NR, see above), we had observed attenuation of threat-related activity in the rostral dmPFC/ACC (Kalisch et al., 2005) while, in the present study, we observed a corresponding interaction in the right anterior insula (see Results, standard analysis). We have meanwhile amassed evidence that the rostral $\mathrm{dmPFC} / \mathrm{ACC}$ is involved in conscious threat appraisal (Kalisch et al., 2006; Mechias et al., 2010) and, in its extreme form, catastrophizing (Raczka et al., 2010). It is therefore conceivable that the use of an explicit immersion comparison condition that promotes negative reflection about the situation in the previous study had boosted rostral dmPFC/ACC responding to threat (T/NR condition), making it in turn sensitive for attenuation by reappraisal ( $\mathrm{T} / \mathrm{R}$ condition). Conversely, in the present study, subjects were simply asked to pay attention to their feelings and reactions in the NR comparison condition. This may have enhanced insula-dependent interoceptive awareness (Craig, 2009) and made the insula a primary neural target of reappraisal in the $\mathrm{T} / \mathrm{R}$ condition.

Unexpectedly, reappraisal also induced left posterior LFC as well as dmPFC/ACC activations with a tonic response profile. While these again speak for reappraisal being a temporally extended process, we have nevertheless not predicted them. IMMO holds that subjects make renewed implementation or enhanced maintenance efforts, if performance monitoring signals insufficient reappraisal success. One could speculate that the current task of detaching from a threat of being shocked was difficult enough to engage such re-iterant processing to some extent. The small effect sizes in our subjective and physiological measures of anxiolysis could be taken to support this idea. The similarity of tonic and linearly decreasing main effects of reappraisal in terms of their anatomical distributions would suggest that subjects repeatedly recurred to retrieving the learned reappraisal material into working memory (implementation). Better previous training or a smaller anxiogenic challenge should then reduce this effect. An 
alternative possibility that we cannot exclude is that the tonic left posterior LFC activation reflects working memory operations that function to continuously maintain the reappraisal material online. In this case, working memory maintenance would commence earlier in a trial than predicted by IMMO (perhaps because strategy implementation was made easy and quick due to prior training in this study) and leave only a monitoring function specifically to the late stage of reappraisal. Better previous training (resulting in easier implementation) should not reduce, but rather enhance, this effect. Use of a better matched control NR condition (see above) should abolish the effect, because in that case both the $\mathrm{R}$ and the NR condition would rely on working memory.

A final potentially interesting observation is that dorsomedial prefrontal reappraisal effects were confined primarily to comparatively posterior sites in the tonic and decreasing main effects (corresponding approximately to supplemental or pre-supplemental motor areas and mid parts of the AACC) but located comparatively more rostrally in the increasing main effect (see Tables 1-3), in a region previously linked with self-referential processing (Amodio and Frith, 2006). In a similar fashion, right anterior LFC activation, that we here observed again to increase over reappraisal episodes, has been associated with self-monitoring (reviewed in Kalisch, 2009). Together, these findings would be in accordance with later processing stages comprising a self-monitoring component that serves to determine reappraisal success and perhaps also the correct application of the given reappraisal strategy.

We would like to emphasize that we do not attempt to downplay the important roles that non-frontal areas may play in

\section{REFERENCES}

Amodio, D. M., and Frith, C. D. (2006). Meeting of minds: the medial frontal cortex and social cognition. Nat. Rev. Neurosci. 7, 268-277.

Buchel, C., Morris, J., Dolan, R. J., and Friston, K. J. (1998). Brain systems mediating aversive conditioning: an event-related fMRI study. Neuron 20, 947-957.

Craig, A. D. (2009). How do you feel now? The anterior insula and human awareness. Nat. Rev. Neurosci. 10, 59-70.

Deveney, C. M., and Pizzagalli, D. A. (2008). The cognitive consequences of emotion regulation: an ERP investigation. Psychophysiology 45, 435-444.

Dillon, D. G., Ritchey, M., Johnson, B. D., and Labar, K. S. (2007). Dissociable effects of conscious emotion regulation strategies on explicit and implicit memory. Emotion 7, 354-365.

Domes, G., Schulze, L., Bottger, M., Grossmann, A., Hauenstein, K., Wirtz, P. H., Heinrichs, M., and Herpertz, S. C. (2010). The neural correlates of sex differences in emotional reactivity and emotion regulation. Hum. Brain Mapp. 31, 758-769.
Duvernoy, H. M. (1999). The Human Brain: Surface, Blood Supply, and Three-Dimensional Sectional Anatomy. Wien: Springer.

Eippert, F., Veit, R., Weiskopf, N., Erb, M., Birbaumer, N., and Anders, S. (2007). Regulation of emotional responses elicited by threat-related stimuli. Hum. Brain Mapp. 28, 409-423.

Erk, S., Mikschl, A., Stier, S., Ciaramidaro, A., Gapp, V., Weber, B., and Walter, H. (2010). Acute and sustained effects of cognitive emotion regulation in major depression. $J$. Neurosci. 30, 15726-15734.

Etkin, A., Egner, T., and Kalisch, R. (2011). Emotional processing in anterior cingulate and medial prefrontal cortex. Trends Cogn. Sci. 15, 85-93.

Friston, K. J., Ashburner, J. T., Kiebel, S. J., Nichols, T. E., and Penny, W. D. (2007). Statistical Parametric Mapping: The Analysis of Functional Brain Images. London: Academic Press.

Goldin, P. R., McRae, K., Ramel, W., and Gross, J. J. (2008). The neural bases of emotion regulation: reappraisal and suppression of negative emotion. Biol. Psychiatry 63, $577-586$.

reappraisal but have chosen to focus this analysis and our theorizing on frontal areas, based on the assumption that these mediate the essential executive process that govern any reappraisal. It may also be useful to point out that IMMO has been developed to better understand the deliberate and effortful regulation of emotions, but we cannot exclude that IMMO might also be applicable to situations of deliberate and effortful emotion generation. While in the context of the current study, the distinction between emotion generation and regulation is straightforward, there may be instances where the distinction is less clear or perhaps impossible to make (see Gross et al., 2011). Notwithstanding this current debate, we believe that delineating the mental processes that construct an experience or behavior of interest is generally more useful than focusing on broad categories such as "generation" and "regulation" or also "emotion" and "cognition" (Etkin et al., 2011).

To conclude, we hope to have contributed to a further characterization of the functional architecture of reappraisal, both at an algorithmic (cognitive processes) and implementational (neural substrates) level. We are confident that a more precise mechanistic account of reappraisal is an important basis for investigating dysfunctionality in emotion regulation in patients and can give important hints as to how to best improve emotion regulation, be it with psychological, pharmacological, or neurotechnological instruments.

\section{ACKNOWLEDGMENTS}

This study was funded by the Deutsche Forschungsgemeinschaft (DFG Emmy Noether group KA1623/3-1 and Transregional DFG Research Unit FOR1328, sub-project 3, KA1623/5-1).

Gross, J. J. (1998). Antecedent- and response-focused emotion regulation: divergent consequences for experience, expression, and phsyiology. J. Pers. Soc. Psychol. 75, 224-237.

Gross, J. J. (2002). Emotion regulation: affective, cognitive, and social consequences. Psychophysiology 39, 281-291.

Gross, J. J., Sheppes, G., and Urry, H. L. (2011). Cognition and emotion lecture at the 2010 SPSP emotion preconference. Cogn. Emot. 25, 765 781.

Gross, J. J., and Thompson, R. A. (2007). "Emotion regulation: conceptual foundations," in Handbook of Emotion Regulation, ed. J. J. Gross (New York: Guilford Press), 3-26.

Hajcak, G., and Nieuwenhuis, S. (2006). Reappraisal modulates the electrocortical response to unpleasant pictures. Cogn. Affect. Behav. Neurosci. 6, 291-297.

Houston, B. K., and Holmes, D. S. (1974). Effect of avoidant thinking and reappraisal for coping with threat involving temporal uncertainty. J. Pers. Soc. Psychol. 30, 382-388.

Kalisch, R. (2009). The functional neuroanatomy of reappraisal: Time matters. Neurosci. Biobehav. Rev. 33, 1215-1226.

Kalisch, R., Wiech, K., Critchley, H. D., and Dolan, R. J. (2006). Levels of appraisal: a medial prefrontal role in high-level appraisal of emotional material. Neuroimage 30, 1458-1466.

Kalisch, R., Wiech, K., Critchley, H. D., Seymour, B., O’Doherty, J. P., Oakley, D. A., Allen, P., and Dolan, R. J. (2005). Anxiety reduction through detachment: subjective, physiological, and neural effects. J. Cogn. Emot. 17, 874-883.

Kim, S. H., and Hamann, S. (2007). Neural correlates of positive and negative emotion regulation. $J$. Cogn. Neurosci. 19, 776-798.

Kross, E., and Ayduk, O. (2008). Facilitating adaptive emotional analysis: distinguishing distanced-analysis of depressive experiences from immersed-analysis and distraction. Pers. Soc. Psychol. Bull. 34, 924-938.

Laux, L., Glanzmann, P., Schaffner, P., and Spielberger, C. D. (1981). Das State-Trait-Angstinventar (Testmappe mit Handanweisung, Fragebogen STAI-G Form X 1 und Fragebogen STAI-G Form $X$ 2). Weinheim: Beltz. 
Mak, A. K., Hu, Z. G., Zhang, J. X., Xiao, Z., and Lee, T. M. (2009). Sex-related differences in neural activity during emotion regulation. Neuropsychologia 47, 2900-2908.

McRae, K., Ochsner, K. N., Mauss, I. B., Gabrieli, J. D. D., and Gross, J. J. (2008). Gender differences in emotion regulation: an fMRI study of cognitive reappraisal. Group Proc. Intergroup Relat. 11, 143-162.

Mechias, M. L., Etkin, A., and Kalisch, R. (2010). A meta-analysis of instructed fear studies: implications for conscious appraisal of threat. Neuroimage 49, 1760-1768.

Moser, J. S., Hajcak, G., Bukay, E., and Simons, R. F. (2006). Intentional modulation of emotional responding to unpleasant pictures: an ERP study. Psychophysiology 43, 292-296.

Moser, J. S., Krompinger, J. W., Dietz, J., and Simons, R. F. (2009). Electrophysiological correlates of decreasing and increasing emotional responses to unpleasant pictures. Psychophysiology 46, 17-27.

Ochsner, K. N., Bunge, S. A., Gross, J. J., and Gabrieli, J. D. (2002). Rethinking feelings: an FMRI study of the cognitive regulation of emotion. $J$. Cogn. Neurosci. 14, 1215-1229.

Ochsner, K. N., Ray, R. D., Cooper, J. C., Robertson, E. R., Chopra, S., Gabrieli, J. D., and Gross, J. J.
(2004). For better or for worse: neural systems supporting the cognitive down- and up-regulation of negative emotion. Neuroimage 23, 483-499.

Phan, K. L., Fitzgerald, D. A., Nathan, P. J., Moore, G. J., Uhde, T. W., and Tancer, M. E. (2005). Neural substrates for voluntary suppression of negative affect: a functional magnetic resonance imaging study. Biol. Psychiatry. 57, 210-219.

Phillips, M. L., Drevets, W. C., Rauch, S. L., and Lane, R. (2003). Neurobiology of emotion perception I: the neural basis of normal emotion perception. Biol. Psychiatry 54, 504-514.

Raczka, K. A., Gartmann, N., Mechias, M. L., Reif, A., Buchel, C., Deckert, J., and Kalisch, R. (2010). A neuropeptide $S$ receptor variant associated with overinterpretation of fear reactions: a potential neurogenetic basis for catastrophizing. Mol. Psychiatry 15, 1045, 1067-1074.

Richards, J. M. (2004). The cognitive consequences of concealing feelings. Curr. Dir. Psychol. Sci. 13, 131-134.

Roseman, I. J., and Smith, C. A. (2001). "Appraisal theory: overview, assumptions, varieties, controversies," in Appraisal Processes in Emotion: Theory, Methods, Research, eds K. R. Scherer, A. Schorr, and T. Johnstone (New York: OUP), 3-19.
Sander, D., Grandjean, D., and Scherer, K. R. (2005). A systems approach to appraisal mechanisms in emotion. Neural. Netw. 18, 317-352.

Scherer, K. R. (2001). "Appraisal considered as a process of multilevel sequential checking," in Appraisal Processes in Emotion: Theory, Methods, Research, eds K. R. Scherer, A. Schorr, and T. Johnstone (New York, NY: Oxford University Press), 92-120.

Spielberger, C. D. (1985). Assessment of state and trait anxiety: conceptual and methodological issues. South. Psychol. 2, 6-16.

Tzourio-Mazoyer, N., Landeau, B., Papathanassiou, D., Crivello, F., Etard, O., Delcroix, N., Mazoyer, B., and Joliot, M. (2002). Automated anatomical labeling of activations in SPM using a macroscopic anatomical parcellation of the MNI MRI single-subject brain. Neuroimage 15, 273-289.

Urry, H. L., van Reekum, C. M., Johnstone, T., Kalin, N. H., Thurow, M. E., Schaefer, H. S., Jackson, C. A., Frye, C. J., Greischar, L. L., Alexander, A. L., and Davidson, R. J. (2006). Amygdala and ventromedial prefrontal cortex are inversely coupled during regulation of negative affect and predict the diurnal pattern of cortisol secretion among older adults. $J$. Neurosci. 26, 4415-4425.
Walter, H., von Kalckreuth, A., Schardt, D., Stephan, A., Goschke, T., and Erk, S. (2009). The temporal dynamics of voluntary emotion regulation. PLoS ONE 4, e6726. doi: 10.1371/journal.pone.0006726

Conflict of Interest Statement: The authors declare that the research was conducted in the absence of any commercial or financial relationships that could be construed as a potential conflict of interest.

Received: 18 May 2011; accepted: 18 August 2011; published online: 06 September 2011.

Citation: Paret C, Brenninkmeyer J, Meyer B, Yuen KSL, Gartmann N, Mechias M-L and Kalisch $R$ (2011) A test for the implementation-maintenance model of reappraisal. Front. Psychology 2:216. doi: 10.3389/fpsyg.2011.00216

This article was submitted to Frontiers in Cognition, a specialty of Frontiers in Psychology.

Copyright (C) 2011 Paret, Brenninkmeyer, Meyer, Yuen, Gartmann, Mechias and Kalisch. This is an open-access article subject to a non-exclusive license between the authors and Frontiers Media SA, which permits use, distribution and reproduction in other forums, provided the original authors and source are credited and other Frontiers conditions are complied with. 